\title{
Optimization design for front-wheel alignment parameters
}

\author{
En-guo Dong ${ }^{\text {a }}$, Yanfei Li ${ }^{\mathrm{b}}$ and Lei Zhang ${ }^{\mathrm{c}}$ \\ Automotive School, Tianjin University of Technology and Education, 300222, Tianjin, China \\ adegzl@sohu.com, b1531543362@qq.com, czldeg@126.com
}

Keywords: Suspension; alignment parameters; optimization design

Abstract. The front-wheel alignment parameters of vehicle are generally decided based on some dynamic performances of suspension system or some empirical equations, however these approaches have some shortage to obtain an excellent overall vehicle performance. A design approach of front-wheel alignment parameters of mining truck is proposed based on vehicle handling and braking stability. The design variables include inclination angle, caster, camber and toe-in. The optimization objective of the minimum braking sideslip and the swing front-wheel angle is solved applying a multi-island genetic algorithm. The optimization results show that the proposed approach of front-wheel alignment parameters synchronously ensures the braking and handling stability.

\section{Introduction}

Design approaches for front-wheel alignment parameters of vehicle are obsolescence nowadays and the alignment parameters are generally calculated based on dynamic performance of suspension system or some empirical equations. Qian Lijun put forward an optimization design model of front-wheel alignment parameter with optimization objective of the minimum error between dynamic camber and ideal camber within the turning scope [1]. Liang Libiao designed the front-wheel camber and kingpin inclination angle with the minimal changes of camber and kingpin inclination angle in a jumping [2]. Guo Wei proposed an optimization method by adjusting the initial alignment parameters to alleviate tire wear in the movement space of a double wishbone independent suspension [3]. The front-wheel alignment parameters were optimized comprehensively considering the performance of suspension system and steering system [4]. Actually the alignment parameters are related to the dynamic performance of braking system and suspension system, we will synchronously consider braking system and suspension system in order to obtain an excellent overall performance of vehicle. Therefore, we propose a new approach to design the front-wheel alignment parameters which synchronously considers suspension and braking system with the optimization objective of the minimum braking sideslip and swing front-wheel angle on bumpy road. The optimization results are solved applying a multi-island genetic algorithm.

\section{Establishment of Alignment Parameter Model}

We have completed the front-wheel alignment parameters design of some mining truck. The related parameters of the mining truck are as follows: no-load mass is $17.2 \mathrm{t}$ and payload is $22 \mathrm{t}$. Front tread, rear tread and wheelbase respectively are $3100 \mathrm{~mm}, 2550 \mathrm{~mm}$ and $3650 \mathrm{~mm}$. Suspension system is candle type suspension for which the longest and shortest lengths of suspension cylinder are $1386 \mathrm{~mm}$ and $1033 \mathrm{~mm}$ respectively, and the full-load length is $1174 \mathrm{~mm}$. In addition, the mining truck consists of an integrated hydraulic steering system and four disc brakes.

In the design, the braking sideslip and the swing front-wheel angle on bumpy road have been analyzed.

The braking process includes two stages. Firstly, the model is accelerated from the stationary state by driving force; and then it is braked by braking force after $4 \mathrm{~s}$, and the braking process is end as the velocity is $0 \mathrm{~km} / \mathrm{h}$.

The requirement of braking force is described in table 1 . 
Table 1 Braking force

\begin{tabular}{|l|c|c|c|c|}
\hline \multirow{2}{*}{ Type } & \multicolumn{2}{|c|}{$\begin{array}{c}\text { Proportion of brake force } \\
\text { and all mass [\%] }\end{array}$} & $\begin{array}{c}\text { Proportion of brake force } \\
\text { on axle [\%] }\end{array}$ \\
\cline { 2 - 5 } & No-load & Full-load & Front axle & Rear axle \\
\hline Mass $<3500 \mathrm{~kg}$ & $\geq 60$ & $\geq 50$ & $\geq 60$ & $\geq 20$ \\
\hline Others & $\geq 60$ & $\geq 50$ & $\geq 60$ & -- \\
\hline
\end{tabular}

In order to make vehicle effectively be brake and prevent wheel to be locked, the braking force need meet Equ.1

$$
\varphi_{s} m g \leq F=m \ddot{x}_{\max } \leq \varphi_{p} m g
$$

where, $\varphi_{s}$-slide coefficient in a braking; $\ddot{x}_{\max }$-the maximum braking deceleration; $\varphi_{p}$-the maximum tire-road friction coefficient.

In bumpy state, the bumpy road with the cosine wave is used with the amplitude of $40 \mathrm{~mm}$. The cosine waves make front wheels move with reverse direction.

\section{Optimization Design for Suspension Alignment Parameters}

Design Model. According to the above analysis, the objective function of front-wheel alignment parameters is shown as Equ. 2

$$
\min f(X)=f_{1}(X)+f_{2}(X)
$$

where, $f_{1}(X)$ is the braking slide; $f_{2}(X)$ is the swing front-wheel angle on bumpy road. $f_{1}(X)=\max s\left(x_{t}\right), \quad f_{2}(X)=\int_{0}^{l} r(x) d x, t$-braking time, $s$-braking sideslip, $l$-the amplitude of wheel bumpy, $r$ - the swing front-wheel angle.

Design variables are $X=\left(x_{1}, x_{2}, x_{3}, x_{4}\right)^{T}$, respectively corresponding to the inclination angle, caster, camber and toe-in. The range of each variable is $[1,12]^{\circ},[1,6]^{\circ},[0.1,1]^{\circ}$ and $[5,15] \mathrm{mm}$ respectively.

The constraints are related to the braking stability and handling stability. In the vehicle dynamic performance, the braking sideslip won't exceed $5 \mathrm{~m} / \mathrm{km}$, and the maximum swing angle of front wheel won't exceed $3^{\circ}$ on bumpy road with the amplitude of $40 \mathrm{~mm}$.

$$
\left\{\begin{array}{l}
f_{1}(X) \leq 5 \\
f_{2}(X) \leq 3
\end{array}\right.
$$

Optimization Algorithm. The multi-island genetic algorithm is usually used to search the global optimal value. In the algorithm, each design point has a fitness value just like other genetic algorithm which is obtained according to objective value and penalty value. A good objective value for each individuals means that the penalty function also has a higher fitness value. The difference of multi-island genetic method and traditional genetic algorithm is that each population is divided into several sub-populations in the multi-island genetic method, also known as the island. Then the traditional genetic algorithm is carried on in each separate sub-population. Some individuals are chosen to cycle "migration" to other island. This kind of operation is as the "migration". There are two parameters to control migration process: immigration interval (the number of offspring after each immigrant) and immigration rate (the percentage of individual immigration).

The traditional genetic algorithm in the multi-island genetic algorithm is used as the followings. 
Code Scheme. The initial input parameters are expressed by binary integer based on 16 characters, in which the character of 16 th is used to judge the condition of plus or minus, and the other characters of 15 are the real code to show the input data. If a 16-bit code is corresponding to the decimal data $Y_{i j}$, the corresponding input data are denoted $\mathrm{A}_{\mathrm{ij}}=\mathrm{Y}_{\mathrm{ij}} / 32767$ (32767 is the largest decimal data expressed by 15 binary code). According to the rule, the initial input parameters are limited at the range of [-1 1], which ensures the same importance for each input parameters.

Fitness Calculation. Individual fitness is decided based on the error, and the larger error represented the smaller individual fitness, as shown in Equ.4.

$$
F_{i}=C-E_{i}
$$

where, $F_{i}$-the individual fitness of the sample of No.i, $C$-constant, and $E_{i}$-sample error between the actual output and the expected output.

Inheritance. After the completion of individual fitness calculation, the individuals of the larger fitness are inherited to next generation. In the actual process, we have selected the individuals of the larger fitness of 3\% at the top is unconditionally passed on to the next generation.

Reproduction. Whether or not the individuals are reproduced is judged based on the individual fitness. The individuals of the larger fitness are reproduced and the individuals of the smaller fitness are eliminated. The reproduction rate is shown as Equ.5

$$
p_{r}=p_{r}^{0}-k_{r 1} \times e^{\left(\frac{-x}{\max }\right)}-k_{r 2} \times e^{\left(\frac{-a v e}{\max }\right)}
$$

where, $P_{r}^{0}$ - the initial reproduction rate; $k_{r 1}, k_{r 2}$-the reproduction coefficients; $x$-the difference between the maximum and the minimum fitness in each generation; ave-the differences of the average fitness of between two successive generations; max-the largest fitness.

Crossover. The crossover rate is dynamically adjusted according to the differences of solution set between every two generation in the genetic process and the similarity of the strings in each generation. The crossover rate is adjusted as Equ. 6

$$
p_{c}=p_{c}^{0}-k_{c 1} \times e^{\left(\frac{-x}{\max }\right)}-k_{c 2} \times e^{\left(\frac{-a v e}{\max }\right)}
$$

where, $P_{c}^{0}$-the initial crossover rate; $k_{c 1}, k_{c 2}$-the crossover coefficients.

Mutation. For the mutation process, the different mutation rates are required according to the individual performance differences in each generation as Equ.7

$$
p_{m}=p_{m}^{0}-k_{m 1} \times e^{\left(\frac{-x}{\max }\right)}-k_{m 2} \times e^{\left(\frac{- \text { ave }}{\max }\right)}
$$

where, $P_{m}^{0}$-the initial mutation rate; $k_{m 1}, k_{m 2}$-the mutation coefficients.

\section{Results Analysis}

The design results of the front wheel alignment parameters are shown in table 2 . In table 2, method (1) is the results based on the proposed model and method (2) is the calculation results according to reference [6]. In addition, the optimization objectives are shown in Fig.1.

Table 2 Design results

\begin{tabular}{|c|c|c|c|c|}
\hline Method & Inclination angle $\left[{ }^{\circ}\right]$ & Caster $\left[{ }^{\circ}\right]$ & Camber $\left[{ }^{\circ}\right]$ & Toe-in[mm] \\
\hline$(1)$ & 7.1 & 1.0 & 1.0 & 13.8 \\
\hline$(2)$ & 3.0 & 4.7 & 0.5 & 3.9 \\
\hline
\end{tabular}




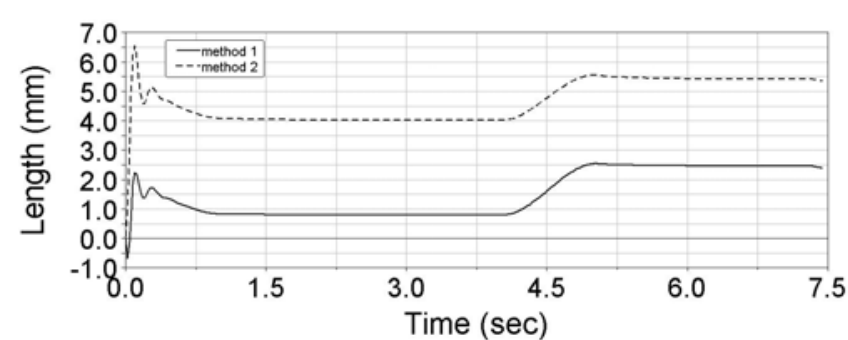

(a) Braking sideslip

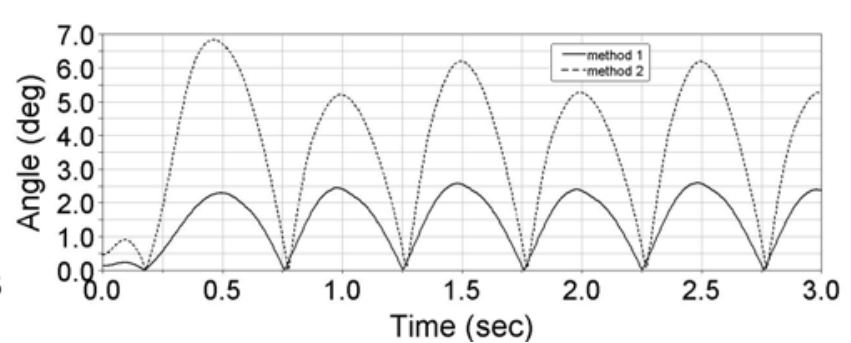

(b) Swing angle of front wheel

Fig.1 Measure value of optimization objective

The design results in the method (2) are better than the results in method (1). As shown in Fig.1, the objective of braking sideslip is $4.2 \mathrm{~mm}$ and the swing front-wheel angle is $6.3^{\circ}$ based on the method (2), while the objective of braking sideslip is $1.9 \mathrm{~mm}$ and the swing front-wheel angle is $2.5^{\circ}$ based on the method (1). The braking sideslip and the swing angle are decreasing by $54.8 \%$ and $60.3 \%$ respectively in the method (1). The reason is in the method (2) the kingpin inclination angle and caster have been calculated based on aligning torque which hasn't taken into account the dynamic characteristic of vehicle on bumpy road. In addition, the variables of camber and toe-in in method (2) have been calculated based on driving sideslip, while in method (1) the alignment parameters of front wheel have been calculated based on the brake sideslip and the swing front-wheel angle on bumpy road.

\section{Summary}

A new design approach of front wheel alignment parameters of mining truck is proposed based on the braking and handling stability of vehicle. The approach makes the design for alignment parameters of front wheel become simple and efficient by analyzing the different working states into the multi-objective optimization model. The optimization results show that the approach of alignment parameters of front wheel overcomes the defects of traditional method. In future, the design for front-wheel alignment parameters should be considered based on suspension system, steering mechanism and brake system to improve the overall vehicle performance.

\section{Acknowledgments}

This work was financially supported by the Chinese National Natural Science Foundation (51005167) and Tianjin University of Technology and Education Science Foundation (KJY14-01).

\section{References}

[1]Qian Lijun, Xiang Shiwei, Zhu Anding, etc. Optimization of Wheel Alignment Parameters of Bus based on Reduction of Tire Wear. Automobile Technology, 2 (2011) 14-17.

[2]Liang Libiao, Liu Jianxiong. Optimization of the Front Wheel Alignment Parameters of FSAE Racing Car. Mechanical Science and Technology, 32 (2013) 231-234.

[3]GUO Wei, HE Ren. Optimization of Suspension Initial Alignment Parameters Based on Reducing Tyre Wear. Journal of Jiangsu University of Science and Technology, 23 (2002) 49-53.

[4]Dong Enguo, Zhang Lei, Shen Yanhua. Research on Front Wheel Alignment Parameters of Auto Based on Second Order Response Surface Model. Automobile Technology, 11 (2007) 22-25.

[5]Guan Xin, Wu Zhenxin, Zhan Jun. A Study on Suspension Modeling for Real-time Vehicle Dynamics Simulation. Automotive Engineering, 29 (2007) 433-436.

[6]Wei Daogao, Zhou Fugeng, Li Lei, etc. Calculation on Front Wheel Kingpin Inclination of Double-wishbone Independent Suspension. Automotive Engineering, 27 (2005) 431-433. 